\title{
Botanical Medicine and Phytochemicals in Healthy Aging and Longevity-Part 2
}

\section{Khara Lucius, ND, FABNO}

\section{Introduction}

In Part 1 of this review, we discussed botanical medicines that may influence either telomeres or the mechanistic target of the rapamycin (mTOR) pathway in healthy aging. In Part 2, we now turn our attention to sirtuin (SIRT) proteins, peroxisome proliferator-activated receptor $\gamma$ coactivator $1-\alpha$ (PGC-1 $\alpha$ ), AMP-activated protein kinase (AMPK) as a mediator of metabolism, and nuclear factor erythroid 2-related factor 2 (Nrf2) as a regulator of oxidative defense. Each of these factors plays a critical role in either minimizing the damage associated with the normal aging process or in supporting the mechanisms that repair that damage.

\section{Sirtuin Proteins}

SIRTs are a family of proteins that chiefly function as deacetylases, enzymes that remove acetyl groups from proteins. SIRTs are evolutionarily conserved from prokaryotes to eukaryotes, with the SIRT family having seven members in mammals (numbered SIRT1-7). SIRTs perform a number of important biological functions, with SIRT1 being the most extensively studied of the group. SIRT1 participates in the formation of chromatin (the material that condenses to form chromosomes), alters gene expression, modulates p53 deacetylation (preventing cellular senescence and suppressing apoptosis), inhibits adipogenesis, deacetylates nuclear factor $\kappa \beta(\mathrm{NF}-\kappa \beta)$, regulates mitochondrial biogenesis, and controls various transcription factors. ${ }^{1}$ The additional SIRT proteins are involved in tumor suppression, regulation of mitosis and cellular proliferation, and DNA repair processes, among other functions. SIRT1, 3, and 4 also play important roles in regulating metabolism in response to dietary changes and nutrient availability. SIRT4, for example, regulates glutamate metabolism in response to amino acid intake. ${ }^{2}$ The potential impact of nutrient availability on lifespan is highlighted by studies of calorie restriction (CR). CR is perhaps one of the best-known interventions to improve lifespan in numerous models, including yeast, nematodes, flies, fish, and rodents (whether or not CR actually extends lifespan in people is unknown at this time). ${ }^{3}$ It is possible that some of the effects of CR are mediated by SIRTs, and levels of most SIRTs appear to be upregulated by $\mathrm{CR} .{ }^{4}$

SIRT expression is correlated with lifespan in several experimental models. SIRT2 overexpression increases lifespan in yeast by up to $70 \%{ }^{5}$ Increased SIRT expression also extends lifespan in a Caenorhabditis elegans (nematode) model, and in Drosophila melanogaster. ${ }^{4}$ In mice, SIRT6 depletion leads to premature aging, degenerative diseases, and early death. ${ }^{6}$ Overexpression of SIRT6, in contrast, reduces insulin-like growth factor 1, promoting improved glucose tolerance and increased lifespan in mice. ${ }^{7}$ Importantly, the effects of SIRTs in lifespan are not necessarily cut and dry. The activity of SIRTs (and deacetylases) is context dependent. The presence of specific stressors may influence SIRT activity. For example, a low to moderate increase in SIRT1 levels may be protective against cardiovascular disease (CVD) in mice by reducing oxidative stress, whereas overexpression of SIRT1 at higher levels actually increases oxidative stress and shortens lifespan. ${ }^{8}$

Fascinatingly, polymorphisms of SIRT genes have recently been detected in humans, and specific genotypes may confer protection against CVD or influence susceptibility to breast cancer. ${ }^{9-11}$ In an Italian study, a specific polymorphism of SIRT3 has been found to be correlated with lifespan in men aged 90 years or older. ${ }^{12}$

Of the natural substances that may influence SIRT activity, resveratrol is certainly the best studied. Interest in resveratrol, the stilbenoid polyphenol found in Japanese knotweed (Fallopia japonica), grapes, red wine, peanuts, and berries, initially arose with the discovery of its antioxidant properties. Since this initial discovery, research on resveratrol has expanded tremendously, and we now have much more information about how this polyphenol exerts its antioxidant activities. Resveratrol inhibits lipid peroxidation and scavenges peroxyl and hydroxyl free radicals, protecting deoxyribose. ${ }^{13}$ Part 1 of this article reviewed the potential effects of resveratrol on mTOR. Although resveratrol may have multiple targets, it is certainly best known for its effects as an activator of SIRT. ${ }^{14}$ Data on resveratrol's impact on SIRTs in model organisms are intriguing.

In a yeast model utilizing Saccharomyces cerevisiae, application of resveratrol resulted in a $70 \%$ increase in lifespan. 
Among three polyphenols tested (resveratrol, butein, and fisetin), resveratrol produced the greatest degree of SIRT2 activation. ${ }^{15}$ In invertebrate models (C. elegans and Drosophila), resveratrol has also been found to increase lifespan in a dosedependent manner, with effects again being mediated by SIRT2. ${ }^{16}$ One of the first studies to demonstrate an effect of resveratrol on lifespan in vertebrates was published in 2006, with Valenzano and Cellerino demonstrating improved longevity and delay of age-related degeneration in fish treated with resveratrol. ${ }^{17}$ From there, research moved on to examine the effects of resveratrol in mammals. At this time, no study has yet demonstrated an effect of resveratrol on lifespan in healthy mammals. Three studies in mice have shown no effect, and one study in rats given either red wine or a resveratrol supplement showed improved vascular aging, but no change to lifespan. ${ }^{18}$ However, in metabolically compromised mammals (mice fed a high-fat diet), resveratrol has been shown to improve lifespan. ${ }^{19}$ Resveratrol also benefits monkeys fed a highfat and high-sugar diet, helping reduce arterial inflammation, adipocyte size, and insulin resistance, while also increasing SIRT1 expression. ${ }^{20,21}$

Of course, the question of greatest interest is whether resveratrol influences SIRTs in people. A handful of small clinical trials shed light on this question.

A 2014 trial by Goh et al. randomized 10 people with type 2 diabetes (T2DM) to receive either $3000 \mathrm{mg}$ resveratrol, or a placebo, every day for 12 weeks. Subjects who took resveratrol compared with placebo had significantly increased skeletal muscle SIRT1 expression $(P=0.016)$. Subjects in the resveratrol group also experienced a significant increase in AMPK expression $(P=0.032)$, an effect we will revisit later. ${ }^{22}$

A 2018 trial by Bo et al. also examined the effects of resveratrol in people with T2DM. One hundred twenty-eight subjects were randomized in a double-blind manner to receive either $500 \mathrm{mg}$ resveratrol daily, $40 \mathrm{mg}$ resveratrol daily, or one placebo capsule daily for 6 months. Patients being treated with insulin were excluded. At the conclusion of the trial, subjects in the $500 \mathrm{mg}$ group had significantly increased SIRT1 levels $(P<0.001)$, whereas subjects in the lower dose and placebo groups did not. Increased SIRT1 levels were also associated with improved serum antioxidant status $(P=0.047){ }^{23}$

A 2017 trial conducted by Mansur et al. randomized healthy middle-aged adults $(N=48)$ to either a calorie-restricted diet (1000 kilocalories daily) or a resveratrol supplement at a dose of $500 \mathrm{mg}$ daily for 30 days. Both of these interventions led to significant increases in SIRT1 expression $(P<0.0001$ for the resveratrol group and $P<0.0001$ for the $\mathrm{CR}$ group). Participants in the CR group also experienced decreases in waist circumference and lipids, effects not seen in the resveratrol group. ${ }^{24}$ The same group also analyzed data from this trial looking for an effect on endogeneous secretory receptor for advanced glycation end products (ESRAGE, a measure of endothelial cell protection). They found that both resveratrol and CR improved ESRAGE gene expression. ${ }^{25}$

The preclinical studies and small-scale clinical trials already discussed highlight some of the potential advantages of re- sveratrol supplementation. Larger scale clinical trials would provide additional information on these benefits. No trials have specifically been conducted looking at human lifespan and resveratrol supplementation.

\section{Peroxisome Proliferator-Activated Receptor $\gamma$ Coactivator 1- $\alpha$}

PGC- $1 \alpha$ is a transcription coactivator (a protein that increases gene transcription without actually binding to DNA) that has wide-ranging effects on cellular energy metabolism, and is a primary regulator of mitochondrial biogenesis. PGC- $1 \alpha$ expression is high in tissue types that have plentiful mitochondria and higher rates of aerobic metabolism. This includes heart tissue, skeletal muscle, brown adipose tissue, kidney tissue, and the brain. In addition to controlling mitochondrial function, PGC- $1 \alpha$ also plays a role in removing reactive oxygen species (ROS), which are the by-products of mitochondrial metabolism. This serves to increase mitochondrial metabolism while also mitigating the effects of resultant ROS on the cell. ${ }^{26,27}$

The initial discovery of PGC- $1 \alpha>20$ years ago came about with detection of its role as a transcription coactivator in adaptive thermogenesis (AT). AT is involved in heat production in response to cold exposure, fever, stressors, and nutrient availability or composition. ${ }^{28}$ AT mediated by brown adipose tissue helps maintain temperature during cold exposure and regulate fever in infection. This process is also important for obesity. Major et al., in their 2007 review of this subject, point out that AT may also be involved when there is a reduction in "energy expenditure beyond what could be predicted from the changes in fat mass or fat-free mass under conditions of standardized physical activity in response to a decrease in energy intake. ${ }^{29}$ In other words, in some individuals, energy restriction (through reduction of calorie intake and increase in physical activity) may lead to a slowing of metabolic rate. In some cases, this loss of energy expenditure may even be enough to compensate for the deficit achieved with diet and exercise. This may render the efforts of some people to lose weight less effective, and make it harder for these individuals to lose weight without rebounding to a particular "set-point."29

Owing to its central function as a regulator of mitochondrial function (as well as a modulator of ROS removal), PGC- $1 \alpha$ is of course very important in the aging process. Mitochondrial function is generally thought to decline with aging. ${ }^{30}$ In mice, aging is associated with a reduction of PGC- $1 \alpha$ expression, which leads to both increased inflammation and insulin resistance. ${ }^{31}$ PGC- $1 \alpha$ also appears to safeguard skeletal muscle from aging-related atrophy. ${ }^{32}$

As discussed in Part 1 of this review, the various mechanisms involved in aging do not act in isolation, and significant overlap and interaction of these factors with each other may occur. SIRT1 activates expression of PGC- $1 \alpha$ by helping maintain PGC- $1 \alpha$ in its active deacetylated form. ${ }^{33-35}$ In addition, telomere dysfunction is associated with reduced levels 
of PGC- $1 \alpha$, and PGC- $1 \alpha$ appears to modulate both telomere length and telomere function. ${ }^{27,36}$

These overlapping functions become important when considering botanical medicines that may influence PGC- $1 \alpha$ activity. In a mouse model, resveratrol has been found to improve PGC-1 $\alpha$ activity in both skeletal muscle and brown adipose tissue (although not in cardiac tissue, suggesting an effect that is potentially tissue specific). ${ }^{37}$ The authors of this mouse study sought to determine whether these effects were mediated by SIRTs. It appears in this case that most of resveratrol's effects on PGC- $1 \alpha$ were indeed mediated by SIRT1. This is in agreement with cell line data, suggesting that resveratrol enhances PGC- $1 \alpha$, but that these effects are abolished when SIRT1 is inhibited. ${ }^{38}$ Animal data indicate that resveratrol may ameliorate kidney damage after hemorrhagic shock, protect against medication-induced hepatotoxicity, and help reduce lung injury related to ischemia, all through modulation of PGC- $1 \alpha$ or the PGC- $1 \alpha /$ SIRT system. ${ }^{39-41}$

Aside from resveratrol, data on the effects of other botanical medicines and phytochemicals on PGC- $1 \alpha$ are preliminary. A single preclinical study has been performed on Lippia sidoides, an aromatic plant from the verbena family used in traditional Brazilian medicine. This study suggested that Lippia may stimulate expression of PGC- $1 \alpha$, promoting an increase in mitochondrial biogenesis. ${ }^{42}$ A single study of Korean red ginseng has also been performed in a mouse model of diabetes. Mice that received treatment with Korean red ginseng powder (for 12 weeks at a dose of $100 \mathrm{mg} / \mathrm{kg}$ once daily through intraperitoneal injection) had improved expression of PGC- $1 \alpha$ compared with mice that received a saline injection. The mice treated with ginseng also had lower levels of the inflammatory markers interleukin (IL)-6, C-reactive protein (CRP), and cyclooxygenase $2(\mathrm{COX}-2){ }^{43}$

Quercetin, an anti-inflammatory flavonoid found in numerous fruits and vegetables, such as onions, citrus, grapes, berries, broccoli, and cherries, is also present in a number of botanical medicine species, ${ }^{44}$ and may work to activate PGC- $1 \alpha$. Table 1 provides a list of quercetin-containing botanical medicines.

In a rat model of hypoxia-induced cognitive decline, quercetin improved expression of PGC- $1 \alpha$ and ameliorated memory impairment. ${ }^{45}$ In an experimental model of neurodegeneration, also in rats, quercetin has been observed to upregulate PGC- $1 \alpha$, improve mitochondrial biogenesis, and decrease

\begin{tabular}{|c|c|}
\hline \multicolumn{2}{|c|}{$\begin{array}{l}\text { Table 1. Quercetin-Containing Botanical } \\
\text { Medicines }\end{array}$} \\
\hline Scientific name & Common name(s) \\
\hline Moringa oleifera & Moringa, drumstick tree \\
\hline Centella asiatica & Gotu kola \\
\hline Hypericum perforatum & St. John's wort \\
\hline Camellia sinensis & Black tea, green tea \\
\hline Vaccinium oxycoccos & Cranberry \\
\hline
\end{tabular}

ROS levels in the rat brain. ${ }^{46}$ And in a mouse model of traumatic brain injury, quercetin upregulates PGC- $1 \alpha$ expression, resulting in improved antioxidant levels and mitochondrial function. ${ }^{47}$

Although there are no clinical trials examining the effects of these phytochemicals or botanical medicines on PGC- $1 \alpha$, human studies do demonstrate that quercetin supplementation improves neuromuscular performance during resistance training exercise, improves endurance and $\mathrm{VO}_{2} \max$, and enhances aerobic exercise performance while increasing markers of mitochondrial biogenesis. ${ }^{48-50}$

\section{AMP-Activated Protein Kinase}

The AMPK pathway works as a key metabolic sensor and is activated under conditions of reduced cellular energy. When cellular adenosine triphosphate (ATP) declines, as may occur when nutrient levels are depleted, or with prolonged exercise, activation of AMPK inhibits anabolism, and increases catabolism to generate additional ATP. ${ }^{51}$ AMPK activation promotes a metabolic shift from fat synthesis to fat oxidation, and increases the uptake of glucose by skeletal muscle. AMPK also acts on a number of transcription factors to inhibit cellular growth, an important effect when cellular nutrient supplies are insufficient. ${ }^{51,52}$

The interplay of the multiple metabolic and aging factors discussed in both parts of this review becomes especially apparent when looking at AMPK. AMPK activates SIRTs as well as Nrf2. ${ }^{53}$ AMPK activation also regulates mitochondrial homeostasis by prompting the destruction and elimination of damaged mitochondria - a process called mitophagy — while stimulating the production of new mitochondria. This effect on mitochondrial biogenesis is mediated by AMPK-dependent stimulation of PGC- $1 \alpha{ }^{51}$ In addition, AMPK controls autophagy by regulating the mTOR signaling. As detailed in Part 1 of this review, autophagy is an important normal mechanism by which intracellular proteins or damaged organelles (such as mitochondria) are removed from the cell. Autophagy prevents cellular necrosis, promotes normal cellular senescence, and protects cells against genomic damage. By helping cells adapt to and respond to stress, autophagy can delay cellular apoptosis and extend the lifespan of the cell. ${ }^{54}$

Appropriate regulation of AMPK, therefore, has farreaching implications for aging, health, and disease. Aging itself may be associated with reductions in AMPK activity or in the AMPK response. ${ }^{55}$ It is thought that a decline in AMPK associated with aging may lead to a number of important metabolic changes, such as increased fat deposition, oxidative stress, and inflammation, and decreased autophagy and insulin sensitivity. ${ }^{53}$ Increased AMPK expression has been found to improve lifespan in a nematode model as well as in Drosophila. ${ }^{56,57}$ Interest in pharmacologic agents that may improve AMPK function has of course been high, and in 2001 metformin was found to be an AMPK activator. ${ }^{52} \mathrm{~A}$ number of plants or plant extracts have also been found to activate AMPK in cell culture and animal studies, including 
resveratrol, berberine, quercetin, genistein, curcumin, ginseng, green tea, and Gynostemma pentaphyllum.

Resveratrol is currently the only substance of those already listed to have been specifically tested for its effects on AMPK in humans, and these studies are mixed, with two showing positive effects, and two showing no impact. In a small trial $(N=11)$ of obese men, resveratrol supplementation at $150 \mathrm{mg}$ given daily in a double-blind crossover manner for 30 days was found to increase AMPK phosphorylation in skeletal muscle, as well as SIRT and PGC- $1 \alpha$ levels (individual $P$-values for these findings were not provided by the authors) ${ }^{58}$ In Goh et al.'s trial, already discussed under SIRTs, 10 participants with T2DM were supplemented with $3000 \mathrm{mg}$ resveratrol, or a placebo, daily for 12 weeks. The resveratrol group experienced a significant increase in skeletal muscle AMPK expression $(P=0.032)$ after supplementation. ${ }^{22}$ Two additional trials did not demonstrate an effect. Yoshino et al. randomized nonobese women $(N=29)$ with normal glucose tolerance to receive either $75 \mathrm{mg}$ resveratrol, or a placebo, once daily for 12 weeks. They found no impact of supplementation on AMPK levels in skeletal muscle or adipose tissue, nor on body weight or composition, or glucose, insulin, lipid, adipokine, CRP, or IL-6 levels. ${ }^{59}$ Williams et al. gave a single dose of resveratrol $(300 \mathrm{mg})$ or a placebo to overweight sedentary men in a randomized cross over double-blind manner. They found no impact of this single dose on AMPK expression in skeletal muscle or adipose tissue, nor on SIRT activity. ${ }^{60}$

These four studies highlight interesting questions about the impact of resveratrol on AMPK. Might resveratrol work differently in normal weight individuals, versus those with existing metabolic challenges, such as obesity or T2DM? Are single doses simply insufficient to produce an effect? Are higher doses more effective than lower doses? Additional human trials would help provide answers to these questions.

Turning to berberine, ginseng, green tea, and G. pentaphyllum, preclinical evidence provides some preliminary information about effects on AMPK.

Berberine is an alkaloid plant compound and one of the main constituents in the root of Coptis chinensis, a plant that has been used in Traditional Chinese Medicine (TCM) to treat diabetes for thousands of years. ${ }^{61}$ Preclinical data indicate berberine may have specific benefits for cardiovascular and hepatic tissue through modulation of AMPK. In cardiomyoblast cells, berberine has been found to not only activate AMPK but also inhibit mTOR. ${ }^{62}$ In an additional cell line study, a hyperglycemia model of cardiomyocyte hypertrophy was used to demonstrate that berberine improved mitochondrial function by increasing mitochondrial biogenesis and improving clearance of damaged mitochondria through autophagy, while also increasing AMPK signaling. In mice on a high-fat diet, berberine increases AMPK phosphorylation in the liver, reducing triglyceride (TG) synthesis and ameliorating hepatic steatosis. ${ }^{63}$ Likewise, in a rat model of nonalcoholic fatty liver disease (NAFLD), supplementation with berberine leads to a reduction in hepatic steatosis through increased expression of AMPK and SIRT3 in the liver. ${ }^{64}$ Clinical trials indicate that berberine helps regulate lipids and blood pressure, and also supports improved blood sugar regulation in people with T2DM. ${ }^{65}$

Ginseng, mentioned previously for its potential effects on PGC-1 $\alpha$, may also exert effects through modulation of AMPK. Ginsenoside Rc, one of the major saponins of Panax ginseng, enhances glucose uptake by mouse muscle fibers by activating AMPK. ${ }^{66}$ An extract of $P$. ginseng C.A. Meyer (Korean red ginseng) has been found to protect mitochondrial function as well as enhance cellular survival by activating AMPK in an iron-induced hepatocyte model of toxicity. ${ }^{67}$ In a mouse model of hepatotoxicity, Korean red ginseng extract also reduces hepatic steatosis through activation of AMPK and SIRT $1 .{ }^{68}$ In addition, in a mouse model of T2DM, an ethanol extract of $P$. ginseng C.A. Meyer improved AMPK phosphorylation in the liver, upregulated expression of skeletal muscle glucose transporters, and decreased hemoglobin A1c (HbA1c), TG, and lipid levels. ${ }^{69}$ Although human trials specifically looking at ginseng's effects on AMPK are not available, clinical trials have found that ginseng lowers blood pressure in people with prehypertension, improves postprandial glucose levels in people with T2DM, and has a hypolipidemic effect. ${ }^{70-72}$

Green tea and its constituents, discussed in Part 1 of this review for their effects on mTOR, may also impact AMPK signaling. In rats fed a high fructose diet, feeding green tea leaves was found to have a number of effects. The fructoserich diet led to elevations of insulin, leptin, cholesterol, and TGs, and green tea mitigated these effects. In fact, in this study, four different types of tea leaves were compared (black, green, oolong, and pu-erh), and green tea and pu-erh tea leaves were found to have the strongest impact on lipids, TGs, insulin, and leptin. These effects were found to be mediated by a significant increase in AMPK phosphorylation as well as a reduction of fatty acid synthase expression. ${ }^{73}$ In mice fed a high-fat diet, several similar effects were seen. Mice were given $0.1 \mathrm{~mL}$ of a $98 \%$ epigallocatechin-3-gallate (EGCG) green tea extract at a dose of $50 \mathrm{mg} / \mathrm{kg}$ per day for 16 weeks. Compared with mice in a control group, mice that received EGCG gained less weight on the high-fat diet, had reduced lipid and TG levels, and had increased levels of adiponectin. In addition to this, EGCG led to increased SIRT expression in the liver and increased AMPK activation. ${ }^{74}$ In obese rats, a 12 week treatment with green tea at a dose of $500 \mathrm{mg} / \mathrm{kg}$ for 5 days weekly also led to favorable alteration in lipid levels, body weight, and fatty acid synthesis. The adipose tissue of rats that received green tea demonstrated increased AMPK expression, which was thought to account for the metabolic benefits seen with supplementation. ${ }^{75}$ Green tea polyphenols have also been shown to reduce NAFLD resulting from a high-fat diet in obese rats, through increased AMPK expression. ${ }^{76}$ Specific human trials of green tea extract for AMPK activation are not currently available, although clinical trials have demonstrated that green tea extract increases leptin and reduces low density lipoprotein levels in overweight or obese women with dyslipidemia, and improves liver function in people with NAFLD. ${ }^{77,78}$ 
Lastly, a handful of preclinical studies have found that G. pentaphyllum may have some activity on AMPK. G. pentaphyllum is a member of the Curcubitaceae family, and grows as a vine producing inedible gourd fruits. G. pentaphyllum contains flavonoids, polysaccharides, and saponin compounds, including gypenoside and dammarane-type saponins. ${ }^{79-82}$ Interestingly, G. pentaphyllum has also been found to contain ginsenoside, although it is not related to the ginseng plants botanically. ${ }^{79}$ G. pentaphyllum saponins have been found to increase AMPK expression in myotube and myoblast cells in culture. ${ }^{82,83}$ In obese mice, eight-week supplementation of a heat-processed $G$. pentaphyllum extract rich in dammarane saponins led to decreased body weight and lipids, and increased AMPK activation in skeletal muscle. ${ }^{84}$

\section{Nuclear Factor Erythroid 2-Related Factor 2}

$\mathrm{Nrf2}$, a transcription factor, is an important central controller of cellular antioxidant responses. Nrf2 is normally sequestered in the cells, and levels of the active molecule generally remain low; the liberated Nrf2 protein is short lived and undergoes rapid degradation, with a half-life of $\sim 20$ minutes ${ }^{85}$ Under stressful conditions, however, Nrf2 accumulates and passes from the cytosol of the cell to the nucleus, where it stimulates several target genes that impact antioxidant status. These include multiple targets involved in phase II detoxification, such as glutathione reductase, glutathione-S-transferase, uridine $\mathrm{S}^{\prime}$-diphospho-glucuronosyltransferase, and heme oxygenase 1. Additional targets include genes involved in phosphorylation, cell cycle regulation (and, therefore, cellular growth and apoptosis), and protein transport. Nrf2 also downregulates specific target genes involved in fatty acid biosynthesis. ${ }^{86}$ Because of these actions, Nrf2 and its downstream targets support detoxification of xenobiotics, modulation of inflammation, and the removal of ROS. Nrf2 may also be involved in the permeability of the blood-brain barrier. ${ }^{87}$

Aging may be associated with several significant changes to Nrf2 function or signaling. Most research conducted in this area has involved rodent models. For example, aging in rats has been found to be associated with significant declines in $\mathrm{Nrf} 2$ levels, leading to reductions in glutathione. ${ }^{88}$ Cardiac tissue from aging rats demonstrates altered Nrf2 signaling. ${ }^{89}$ Agerelated decline in Nrf2 function may also be related to neurodegenerative changes. ${ }^{90}$ Additional support for the importance of Nrf2 function in aging comes from lifespan studies. Mice with genetic changes leading to increased Nrf2 activation have an extended lifespan. ${ }^{91}$ Similar effects are seen in other experimental models, such as Drosophila and C. elegans. ${ }^{92,93}$

Astragalus membranaceous, discussed in Part 1 of this review for its potential impact on telomeres, may also exert beneficial effects through upregulation of Nrf2. Long considered a Qi tonifier and adaptogen in TCM, A. membranaceous contains a number of medicinal compounds, including saponins, flavonoids, and 14 separate polysaccharides. ${ }^{94} \mathrm{~A}$. membranaceous polysaccharides have been found to extend healthy lifespan of the nematode C. elegans. ${ }^{95}$ A. membranaceous polysaccharides also increase antioxidants such as superoxide dismutase (SOD), catalase, and glutathione peroxidase in a mouse model of aging. ${ }^{96}$

In a cellular model of oxidative injury, A. membranaceous polysaccharides not only promoted expression of SOD and catalase, they also activated the Nrf2 pathway; in fact, this promotion of Nrf2 activity was thought to be the key driver of increased antioxidant expression. ${ }^{97}$ In a rat model of arthritis, A. membranaceous polysaccharides worked to modulate the Nrf2 pathway, leading to decreased inflammation, reduced ROS, and improved cardiac function. ${ }^{98}$ A. membranaceous polysaccharides have also been found to improve Nrf2 levels, cardiac function, and antioxidant status in a rat model of autoimmune disease. ${ }^{99}$ Human trials specifically demonstrating an impact of $A$. membranaceous or its compounds on $\mathrm{Nrf} 2$ are not available at this time.

Sulforaphane (SFN), the isothiocyanate phytochemical found in cruciferous plants, is considered a potent inducer of Nrf2. ${ }^{85}$ SFN may be obtained from cabbages, broccoli, kale, and cauliflower, with broccoli seeds and broccoli sprouts yielding especially high levels. Support for SFN's effects on Nrf2 comes from both cell line study and animal models. For example, in a cell model of Parkinson's disease, SFN was protective against neurotoxin-induced damages through upregulation of Nrf2. ${ }^{100}$ Cell line data also indicate that increased expression of Nrf2 promoted by SFN may protect bone tissue from disease by reducing osteoclastogenesis. ${ }^{101}$ In mice with experimentally induced atopic dermatitis, SFN increases expression of $\mathrm{Nrf} 2$ and heme oxygenase 1, conferring protection against oxidation and leading to decreased symptoms. ${ }^{102}$ SFN has also been shown to protect mice from hyperoxia-induced acute lung injury, with these effects being dependent on activation of Nrf2. ${ }^{103} \mathrm{SFN}$ also alleviates experimentally induced memory impairment in mice through Nrf2 activation. ${ }^{104}$ And in the retinas of rats with diabetes, treatment with SFN increased Nrf2 levels, improved antioxidant status, and reduced inflammatory cytokines, including tumor necrosis factor- $\alpha$ (TNF- $\alpha$ ), IL-6, and IL1 $\beta .{ }^{105}$ As with the majority of the plants in this review, these specific cellular effects have not been studied in humans to date. However, it should be noted that human studies demonstrate that SFN improves liver function in men with fatty liver by reducing oxidative stress, and decreases serum insulin and homeostasis model of assessment of insulin resistance in people with T2DM. ${ }^{106,107}$

Olive leaf extract (OLE) is another botanical medicine that may impact Nrf2 function. Olive leaves are rich in multiple phenolic compounds, such as flavonols, catechin, flavones (including luteolin), oleoside, and glycosides. ${ }^{108}$ A cell study utilizing endothelial progenitor cells from healthy volunteers demonstrated that oleuropein (a polyphenol found in OLE) protected cells from senescence induced by angiotensin, reduced ROS formation, and also increased cellular proliferation and telomerase activity, with these effects being dependent on an activation in Nrf2 as well as a resultant increase in heme oxygenase $1 .{ }^{109}$ In rats pretreated with OLE before receiving 
cyclophosphamide to induce kidney injury, OLE improved markers of kidney function and upregulated $\mathrm{Nrf} 2$ and heme oxygenase 1. It also reduced several markers of inflammation, including NF- $\kappa \beta$, caspase- 3 , and cytochrome $c{ }^{110}$ In an additional rat study, hydrochloric acid and ethanol were used to induce gastritis and changes to gastric mucosa, and pretreatment with OLE at $100 \mathrm{mg} / \mathrm{kg}$ was found to prevent some damage, whereas pretreatment with OLE at $300 \mathrm{mg} / \mathrm{kg}$ completely prevented gastritis. These effects were achieved through upregulation of Nrf2, enhanced antioxidant production, and a reduction in lipid peroxidation. OLE also led to a decrease in several markers of inflammation in the gastric mucosa, including NF- $\kappa \beta$, TNF- $\alpha$, COX-2, and IL1 $\beta .{ }^{111}$ Clinical trials of OLE have demonstrated its ability to lower lipids and TGs, reduce blood pressure, positively affect vascular function, and improve insulin sensitivity and glucose homeostasis. ${ }^{12-116}$ Despite this, no studies have been conducted in people specifically evaluating the impact of OLE on Nrf2.

\section{Conclusions}

Aging healthfully involves not just a focus on lifespan itself, but also on healthspan. In Parts 1 and 2 of this review, we have delved into some of the evidence on botanical medicines and phytochemicals that may influence many of the mechanisms involved in aging. Most of these data are preclinical, and as discussed in Part 1, this review mainly discusses mechanistic theories of aging. Our end goal as clinicians is to look at how this may translate to therapeutic outcomes and impact the health of our patients on an everyday basis. Because the data in these areas are largely preclinical at this time, there are numerous opportunities to conduct clinical research. Human study would help bridge the gap between preclinical models and clinical science, allowing us to better understand how these mechanisms may translate into the promotion of healthful longevity for the people we work with.

\section{References}

1. Shoba B, Lwin ZM, Ling LS, et al. Function of sirtuins in biological tissues. Anat Rec 2009;292:536-543.

2. Michan S, Sinclair D. Sirtuins in mammals: Insights into their biological function. Biochem J 2007;404:1-13.

3. Heilbronn LK, Ravussin E. Calorie restriction and aging: Review of the literature and implications for studies in humans. Am J Clin Nutr 2003;78: 361-369.

4. Grabowska W, Sikora E, Bielak-Zmijewska A. Sirtuins, a promising target in slowing down the ageing process. Biogerontology 2017;18:447-476.

5. Kaeberlein M, McVey M, Guarente L. The SIR2/3/4 complex and SIR2 alone promote longevity in Saccharomyces cerevisiae by two different mechanisms. Genes Dev 1999;13:2570-2580.

6. Mostoslavsky R, Chua KF, Lombard DB, et al. Genomic instability and aging-like phenotype in the absence of mammalian SIRT6. Cell 2006; 124:315-329.
7. Kanfi Y, Naiman S, Amir G, et al. The sirtuin SIRT6 regulates lifespan in male mice. Nature 2012;483:218-221.

8. Alcendor RR, Gao S, Zhai P, et al. SIRT1 regulates aging and resistance to oxidative stress in the heart. Circ Res 2007;100:1512-1521.

9. Nasiri M, Rauf M, Kamfiroozie H, et al. SIRT1 gene polymorphisms associated with decreased risk of atherosclerotic coronary artery disease. Gene 2018;672:16-20.

10. Mohtavinejad N, Nakhaee A, Harati H, et al. SIRT1 gene is associated with cardiovascular disease in the Iranian population. Egypt J Med Hum Genet 2015;16:117-122.

11. Rizk SM, Shahin NN, Shaker OG. Association between SIRT1 gene polymorphisms and breast cancer in Egyptians. PLoS One 2016;11:e151901.

12. Bellizzi D, Rose G, Cavalcante $P$, et al. A novel VNTR enhancer within the SIRT3 gene, a human homologue of SIR2, is associated with survival at oldest ages. Genomics 2005;85:258-263.

13. Murcia MA, Martínez-Tomé M. Antioxidant activity of resveratrol compared with common food additives. J Food Prot 2001;64:379-384.

14. Weiskirchen S, Weiskirchen R. Resveratrol: How much wine do you have to drink to stay healthy? Adv Nutr 2016;7:706-718.

15. Howitz KT, Bitterman KJ, Cohen HY, et al. Small molecule activators of sirtuins extend Saccharomyces cerevisiae lifespan. Nature 2003;425: 191-196.

16. Wood JG, Rogina B, Lavu S, et al. Sirtuin activators mimic caloric restriction and delay ageing in metazoans. Nature 2004;430:686-689.

17. Valenzano DR, Cellerino A. Resveratrol and the pharmacology of aging: A new vertebrate model to validate an old molecule. Cell Cycle 2006;5: 1027-1032.

18. Bhullar KS, Hubbard BP. Lifespan and healthspan extension by resveratrol. Biochim Biophys Acta 2015;1852:1209-1218.

19. Baur JA, Pearson KJ, Price NL, et al. Resveratrol improves health and survival of mice on a high-calorie diet. Nature 2006;444:337-342.

20. Mattison JA, Wang M, Bernier $M$, et al. Resveratrol prevents high fat/ sucrose diet-induced central arterial wall inflammation and stiffening in nonhuman primates. Cell Metab 2014;20:183-190.

21. Jimenez-Gomez Y, Mattison JA, Pearson KJ, et al. Resveratrol improves adipose insulin signaling and reduces the inflammatory response in adipose tissue of rhesus monkeys on high-fat, high-sugar diet. Cell Metab 2013;18:533-545.

22. Goh KP, Lee HY, Lau DP, et al. Effects of resveratrol in patients with type 2 diabetes mellitus on skeletal muscle SIRT1 expression and energy expenditure. Int J Sport Nutr Exerc Metab 2014;24:2-13.

23. Bo $\mathrm{S}$, Togliatto $\mathrm{G}$, Gambino R, et al. Impact of sirtuin-1 expression on H3K56 acetylation and oxidative stress: A double-blind randomized controlled trial with resveratrol supplementation. Acta Diabetol 2018;55:331340 .

24. Mansur AP, Roggerio A, Goes MFS, et al. Serum concentrations and gene expression of sirtuin 1 in healthy and slightly overweight subjects after caloric restriction or resveratrol supplementation: A randomized trial. Int J Cardiol 2017;227:788-794.

25. Roggerio A, Strunz CMC, Pacanaro AP, et al. Gene expression of sirtuin1 and endogenous secretory receptor for advanced glycation end products in healthy and slightly overweight subjects after caloric restriction and resveratrol administration. Nutrients 2018;10:7.

26. Puigserver $\mathrm{P}, \mathrm{Wu} \mathrm{Z}$, Park $\mathrm{CW}$, et al. A cold-inducible coactivator of nuclear receptors linked to adaptive thermogenesis. Cell 1998;92:829-839.

27. Austin S, St-Pierre J. PGC1 $\alpha$ and mitochondrial metabolism-emerging concepts and relevance in ageing and neurodegenerative disorders. J Cell Sci 2012;125:4963-4971.

28. Yang X, Ruan H-B. Neuronal Control of Adaptive thermogenesis. Front Endocrinol (Lausanne) 2015;6:149. 
29. Major GC, Doucet E, Trayhurn $P$, et al. Clinical significance of adaptive thermogenesis. Int J Obes 2007;31:204-212.

30. Anderson R, Prolla T. PGC- $1 \alpha$ in aging and anti-aging interventions. Biochim Biophys Acta 2009;1790:1059-1066.

31. Sczelecki S, Besse-Patin A, Abboud A, et al. Loss of Pgc-1 $\alpha$ expression in aging mouse muscle potentiates glucose intolerance and systemic inflammation. Am J Physiol Endocrinol Metab 2014;306:E157-E167.

32. Kang C, Ji LL. Role of PGC- $1 \alpha$ in muscle function and aging. J Sport Heal Sci 2013;2:81-86.

33. Zhou Y, Wang S, Li Y, et al. SIRT1/PGC- $1 \alpha$ signaling promotes mitochondrial functional recovery and reduces apoptosis after intracerebral hemorrhage in rats. Front Mol Neurosci 2017;10:443.

34. Amat R, Planavila A, Chen SL, et al. SIRT1 controls the transcription of the peroxisome proliferator-activated receptor-gamma Co-activator-1alpha (PGC-1alpha) gene in skeletal muscle through the PGC-1alpha autoregulatory loop and interaction with MyoD. J Biol Chem 2009;284:21872-21880.

35. Rodgers JT, Lerin $C$, Gerhart-Hines $Z$, et al. Metabolic adaptations through the PGC-1 $\alpha$ and SIRT1 pathways. FEBS Lett 2008;582:46-53.

36. Xiong S, Patrushev N, Forouzandeh F, et al. PGC- $1 \alpha$ modulates telomere function and DNA damage in protecting against aging-related chronic diseases. Cell Rep 2015;12:1391-1399.

37. Lagouge M, Argmann C, Gerhart-Hines Z, et al. Resveratrol improves mitochondrial function and protects against metabolic disease by activating SIRT1 and PGC-1alpha. Cell 2006;127:1109-1122.

38. Imamura $\mathrm{H}$, Nagayama $\mathrm{D}$, Ishihara $\mathrm{N}$, et al. Resveratrol attenuates triglyceride accumulation associated with upregulation of SIRT1 and lipoprotein lipase in 3T3-L1 adipocytes. Mol Genet Metab Reports 2017;12:44-50.

39. Yeh DY-W, Fu YH, Yang Y-C, et al. Resveratrol alleviates lung ischemia and reperfusion-induced pulmonary capillary injury through modulating pulmonary mitochondrial metabolism. Transplant Proc 2014;46:1131-1134.

40. Nicoletti NF, Rodrigues-Junior V, Santos AA, et al. Protective effects of resveratrol on hepatotoxicity induced by isoniazid and rifampicin via SIRT1 modulation. J Nat Prod 2014;77:2190-2195.

41. Wang H, Guan $Y$, Karamercan MA, et al. Resveratrol rescues kidney mitochondrial function following hemorrhagic shock. Shock 2015;44:173180.

42. Rajgopal A, Burns C, Rebhun J. Lippia sidoides stimulates mitochondrial biogenesis by elevating cAMP levels. Planta Med 2013;79:PN65.

43. Park J-K, Shim J-Y, Cho A-R, et al. Korean red ginseng protects against mitochondrial damage and intracellular inflammation in an animal model of type 2 diabetes mellitus. J Med Food 2018;21:544-550.

44. Anand David AV, Arulmoli R, Parasuraman S. Overviews of biological importance of quercetin: A bioactive flavonoid. Pharmacogn Rev 2016;10: $84-89$.

45. Liu P, Zou D, Yi L, et al. Quercetin ameliorates hypobaric hypoxiainduced memory impairment through mitochondrial and neuron function adaptation via the PGC- $1 \alpha$ pathway. Restor Neurol Neurosci 2015;33:143157.

46. Sharma DR, Sunkaria A, Wani WY, et al. Quercetin protects against aluminium induced oxidative stress and promotes mitochondrial biogenesis via activation of the PGC- $1 \alpha$ signaling pathway. Neurotoxicology 2015;51:116-137.

47. Li X, Wang H, Gao Y, et al. Quercetin induces mitochondrial biogenesis in experimental traumatic brain injury via the PGC- $1 \alpha$ signaling pathway. Am J Transl Res 2016;8:3558-3566.

48. Patrizio F, Ditroilo M, Felici F, et al. The acute effect of quercetin on muscle performance following a single resistance training session. Eur J Appl Physiol 2018;118:1021-1031.

49. Davis JM, Carlstedt CJ, Chen S, et al. The dietary flavonoid quercetin increases VO2max and endurance capacity. Int J Sport Nutr Exerc Metab 2010;20:56-62.
50. Nieman DC, Williams AS, Shanely RA, et al. Quercetin's influence on exercise performance and muscle mitochondrial biogenesis. Med Sci Sports Exerc 2010;42:338-345.

51. Mihaylova MM, Shaw RJ. The AMPK signalling pathway coordinates cell growth, autophagy and metabolism. Nat Cell Biol 2011;13:1016-1023.

52. Hardie DG. AMPK: A target for drugs and natural products with effects on both diabetes and cancer. Diabetes 2013;62:2164-2172.

53. Salminen A, Kaarniranta K. AMP-activated protein kinase (AMPK) controls the aging process via an integrated signaling network. Ageing Res Rev 2012;11:230-241.

54. Mariño G, Niso-Santano M, Baehrecke EH, et al. Self-consumption: The interplay of autophagy and apoptosis. Nat Rev Mol Cell Biol 2014;15:81-94.

55. Reznick RM, Zong H, Li J, et al. Aging-associated reductions in AMPactivated protein kinase activity and mitochondrial biogenesis. Cell Metab 2007;5:151-156.

56. Funakoshi $M$, Tsuda $M$, Muramatsu $K$, et al. A gain-of-function screen identifies WDB and LKB1 as lifespan-extending genes in Drosophila. Biochem Biophys Res Commun 2011;405:667-672.

57. Curtis R, O'Connor G, DiStefano PS. Aging networks in Caenorhabditis elegans: AMP-activated protein kinase (AAK-2) links multiple aging and metabolism pathways. Aging Cell 2006;5:119-126.

58. Timmers S, Konings E, Bilet L, et al. Calorie restriction-like effects of 30 days of Resveratrol (resVida ${ }^{\mathrm{TM}}$ ) supplementation on energy metabolism and metabolic profile in obese humans. Cell Metab 2011;14:612-622.

59. Yoshino J, Conte C, Fontana L, et al. Resveratrol supplementation does not improve metabolic function in nonobese women with normal glucose tolerance. Cell Metab 2012;16:658-664.

60. Williams CB, Hughes MC, Edgett BA, et al. An examination of resveratrol's mechanisms of action in human tissue: Impact of a single dose in vivo and dose responses in skeletal muscle ex vivo. PLoS One 2014;9 e102406.

61. Yin J, Zhang H, Ye J. Traditional Chinese medicine in treatment of metabolic syndrome. Endocr Metab Immune Disord Drug Targets 2008;8: 99-111.

62. Qing Y, Dong X, Hongli L, et al. Berberine promoted myocardial protection of postoperative patients through regulating myocardial autophagy. Biomed Pharmacother 2018;105:1050-1053.

63. Zhu X, Bian H, Wang L, et al. Berberine attenuates nonalcoholic hepatic steatosis through the AMPK-SREBP-1c-SCD1 pathway. Free Radic Biol Med 2019;141:192-204

64. Zhang Y, Deng Y, Tang K, et al. Berberine Ameliorates high-fat dietinduced non-alcoholic fatty liver disease in rats via activation of SIRT3/ AMPK/ACC pathway. Curr Med Sci 2019;39:37-43.

65. Lan J, Zhao Y, Dong F, et al. Meta-analysis of the effect and safety of berberine in the treatment of type 2 diabetes mellitus, hyperlipemia and hypertension. J Ethnopharmacol 2015;161:69-81.

66. Lee M-S, Hwang J-T, Kim S, et al. Ginsenoside Rc, an active component of Panax ginseng, stimulates glucose uptake in $\mathrm{C} 2 \mathrm{C} 12$ myotubes through an AMPK-dependent mechanism. J Ethnopharmacol 2010;127:771-776.

67. Dong G-Z, Jang EJ, Kang SH, et al. Red ginseng abrogates oxidative stress via mitochondria protection mediated by LKB1-AMPK pathway. BMC Complement Altern Med 2013;13:64.

68. Han JY, Lee S, Yang JH, et al. Korean red ginseng attenuates ethanolinduced steatosis and oxidative stress via AMPK/Sirt1 activation. J Ginseng Res 2015;39:105-115.

69. Kang O-H, Shon M-Y, Kong R, et al. Anti-diabetic effect of black ginseng extract by augmentation of AMPK protein activity and upregulation of GLUT2 and GLUT4 expression in $\mathrm{db} / \mathrm{db}$ mice. BMC Complement Altern Med 2017:17:341.

70. Cha TW, Kim M, Kim M, et al. Blood pressure-lowering effect of Korean red ginseng associated with decreased circulating Lp-PLA 2 activity and 
lysophosphatidylcholines and increased dihydrobiopterin level in prehypertensive subjects. Hypertens Res 2016;39:449-456.

71. Oh MR, Park SH, Kim SY, et al. Postprandial glucose-lowering effects of fermented red ginseng in subjects with impaired fasting glucose or type 2 diabetes: A randomized, double-blind, placebo-controlled clinical trial. BMC Complement Altern Med 2014;14:237.

72. Kim SH, Park KS. Effects of Panax ginseng extract on lipid metabolism in humans. Pharmacol Res 2003;48:511-513.

73. Huang H-C, Lin J-K. Pu-erh tea, green tea, and black tea suppresses hyperlipidemia, hyperleptinemia and fatty acid synthase through activating AMPK in rats fed a high-fructose diet. Food Funct 2012;3:170-177.

74. Santamarina AB, Oliveira JL, Silva FP, et al. Green tea extract rich in epigallocatechin-3-gallate prevents fatty liver by AMPK activation via LKB1 in mice fed a high-fat diet. PLoS One 2015;10:e0141227.

75. Rocha A, Bolin AP, Cardoso CAL, et al. Green tea extract activates AMPK and ameliorates white adipose tissue metabolic dysfunction induced by obesity. Eur J Nutr 2016;55:2231-2244.

76. Tan Y, Kim J, Cheng J, et al. Green tea polyphenols ameliorate nonalcoholic fatty liver disease through upregulating AMPK activation in high fat fed Zucker fatty rats. World J Gastroenterol 2017;23:3805.

77. Huang LH, Liu CY, Wang LY, et al. Effects of green tea extract on overweight and obese women with high levels of low density-lipoproteincholesterol (LDL-C): A randomised, double-blind, and cross-over placebocontrolled clinical trial. BMC Complement Altern Med 2018;18:294.

78. Sakata R, Nakamura T, Torimura T, et al. Green tea with high-density catechins improves liver function and fat infiltration in non-alcoholic fatty liver disease (NAFLD) patients: A double-blind placebo-controlled study. Int J Mol Med 2013;32:989-994.

79. Tsai YC, Lin CL, Chen BH. Preparative chromatography of flavonoids and saponins in Gynostemma pentaphyllum and their antiproliferation effect on hepatoma cell. Phytomedicine 2010;18:2-10.

80. Wang Z, Zhao X, Liu X, et al. Anti-diabetic activity evaluation of a polysaccharide extracted from Gynostemma pentaphyllum. Int J Biol Macromol 2019;126:209-214.

81. Tsui K-C, Chiang T-H, Wang J-S, et al. Flavonoids from Gynostemma pentaphyllum exhibit differential induction of cell cycle arrest in $\mathrm{H} 460$ and A549 cancer cells. Molecules 2014;19:17663-17681

82. Nguyen PH, Gauhar R, Hwang SL, et al. New dammarane-type glucosides as potential activators of AMP-activated protein kinase (AMPK) from $G y$ nostemma pentaphyllum. Bioorg Med Chem 2011;19:6254-6260.

83. Ha TKQ, Pham HTT, Cho HM, et al. 12,23-Dione dammarane triterpenes from Gynostemma longipes and their muscle cell proliferation activities via activation of the AMPK pathway. Sci Rep 2019;9:1186.

84. Gauhar R, Hwang S-L, Jeong S-S, et al. Heat-processed Gynostemma pentaphyllum extract improves obesity in ob/ob mice by activating AMPactivated protein kinase. Biotechnol Lett 2012;34:1607-1616.

85. Houghton CA, Fassett RG, Coombes JS. Sulforaphane and other nutrigenomic Nrf2 activators: Can the Clinician's expectation be matched by the reality? Oxid Med Cell Longev 2016:7857186.

86. Matzinger M, Fischhuber K, Heiss EH. Activation of Nrf2 signaling by natural products-can it alleviate diabetes? Biotechnol Adv 2018;36:17381767.

87. Zhang M, An C, Gao Y, et al. Emerging roles of Nrf2 and phase II antioxidant enzymes in neuroprotection. Prog Neurobiol 2013;100:30-47.

88. Suh JH, Shenvi SV, Dixon BM, et al. Decline in transcriptional activity of Nrf2 causes age-related loss of glutathione synthesis, which is reversible with lipoic acid. Proc Natl Acad Sci U S A 2004;101:3381-3386.

89. Gounder SS, Kannan S, Devadoss D, et al. Impaired transcriptional activity of Nrf2 in age-related myocardial oxidative stress is reversible by moderate exercise training. PLoS One 2012;7:e45697.
90. Li Y, Zhao X, Hu Y, et al. Age-associated decline in Nrf2 signaling and associated mtDNA damage may be involved in the degeneration of the auditory cortex: Implications for central presbycusis. Int J Mol Med 2018;42:3371-3385.

91. Singh SP, Niemczyk M, Saini D, et al. Disruption of the mGSTA4 gene increases life span of C57BL mice. J Gerontol A Biol Sci Med Sci 2010;65:14-23

92. Sykiotis GP, Bohmann D. Keap1/Nrf2 signaling regulates oxidative stress tolerance and lifespan in Drosophila. Dev Cell 2008;14:76-85.

93. Tullet JMA, Green JW, Au C, et al. The SKN-1/Nrf2 transcription factor can protect against oxidative stress and increase lifespan in C. elegans by distinct mechanisms. Aging Cell 2017;16:1191-1194.

94. Liu P, Zhao H, Luo Y. Anti-aging implications of Astragalus membranaceus (Huangqi): A well-known chinese tonic. Aging Dis 2017;8:868-886.

95. Wang N, Liu J, Xie F, et al. miR-124/ATF-6, a novel lifespan extension pathway of Astragalus polysaccharide in Caenorhabditis elegans. J Cell Biochem 2015;116:242-251.

96. Li X-T, Zhang Y-K, Kuang H-X, et al. Mitochondrial protection and antiaging activity of Astragalus polysaccharides and their potential mechanism. Int J Mol Sci 2012;13:1747-1761.

97. Han J, Guo D, Sun XY, et al. Repair effects of astragalus polysaccharides with different molecular weights on oxidatively damaged HK-2 cells. Sci Rep 2019;9:9871.

98. Sun Y, Liu J, Wan L, et al. Improving effects of astragalus polysaccharides on cardiac function via Keap1/Nrf2-ARE signal pathway in adjuvant arthritis rats. Chin Herb Med 2016;8:143-153.

99. Wang F, Lui J, Ye Y-F, et al. Astragalus polysaccharides improved the cardiac function in Sjögren's syndrome model rats based on keap 1-Nrf2/ ARE signaling pathway: A mechanism exploration. Chin J Integr Tradit West Med 2014;34:566-574

100. Bao B, Zhang M-Q, Chen Z-Y, et al. Sulforaphane prevents PC12 cells from oxidative damage via the Nrf2 pathway. Mol Med Rep 2019;19:48904896.

101. Xue $\mathrm{P}, \mathrm{Hu} \mathrm{X}$, Powers $\mathrm{J}$, et al. CDDO-Me, sulforaphane and tBHQ attenuate the RANKL-induced osteoclast differentiation via activating the NRF2-mediated antioxidant response. Biochem Biophys Res Commun 2019;511:637-643.

102. Wu W, Peng G, Yang F, et al. Sulforaphane has a therapeutic effect in an atopic dermatitis murine model and activates the Nrf2/HO-1 axis. Mol Med Rep 2019;20:1761-1771.

103. Cho H-Y, Miller-DeGraff L, Blankenship-Paris T, et al. Sulforaphane enriched transcriptome of lung mitochondrial energy metabolism and provided pulmonary injury protection via Nrf2 in mice. Toxicol Appl Pharmacol 2019;364:29-44.

104. Subedi L, Cho KH, Park YU, et al. Sulforaphane-enriched broccoli sprouts pretreated by pulsed electric fields reduces neuroinflammation and ameliorates scopolamine-induced amnesia in mouse brain through its antioxidant ability via Nrf2-HO-1 activation. Oxid Med Cell Longev 2019;2019:3549274.

105. Li S, Yang H, Chen X. Protective effects of sulforaphane on diabetic retinopathy: Activation of the Nrf2 pathway and inhibition of NLRP3 inflammasome formation. Exp Anim 2019;68:221-231.

106. Kikuchi M, Ushida Y, Shiozawa $\mathrm{H}$, et al. Sulforaphane-rich broccoli sprout extract improves hepatic abnormalities in male subjects. World $\mathrm{J}$ Gastroenterol 2015;21:12457-12467.

107. Bahadoran Z, Tohidi M, Nazeri $P$, et al. Effect of broccoli sprouts on insulin resistance in type 2 diabetic patients: A randomized double-blind clinical trial. Int J Food Sci Nutr 2012;63:767-771.

108. Žuntar I, Putnik P, Bursać Kovačević D, et al. Phenolic and antioxidant analysis of olive leaves extracts (Olea europaea L.) obtained by high voltage electrical discharges (HVED). Foods 2019;8:248. 
109. Parzonko A, Czerwińska ME, Kiss AK, et al. Oleuropein and oleacein may restore biological functions of endothelial progenitor cells impaired by angiotensin II via activation of Nrf2/heme oxygenase-1 pathway. Phytomedicine 2013;20:1088-1094.

110. ALHaithloul HAS, Alotaibi MF, Bin-Jumah M, et al. Olea europaea leaf extract up-regulates Nrf2/ARE/HO-1 signaling and attenuates cyclophosphamide-induced oxidative stress, inflammation and apoptosis in rat kidney. Biomed Pharmacother 2019;111:676-685.

111. Al-Quraishy S, Othman MS, Dkhil MA, et al. Olive (Olea europaea) leaf methanolic extract prevents $\mathrm{HCl}$ /ethanol-induced gastritis in rats by attenuating inflammation and augmenting antioxidant enzyme activities. Biomed Pharmacother 2017;91:338-349.

112. Susalit E, Agus N, Effendi I, et al. Olive (Olea europaea) leaf extract effective in patients with stage-1 hypertension: Comparison with Captopril. Phytomedicine 2011;18:251-258.

113. Lockyer S, Corona G, Yaqoob P, et al. Secoiridoids delivered as olive leaf extract induce acute improvements in human vascular function and reduction of an inflammatory cytokine: A randomised, double-blind, placebocontrolled, cross-over trial. Br J Nutr 2015;114:75-83.
114. Lockyer S, Rowland I, Spencer JPE, et al. Impact of phenolic-rich olive leaf extract on blood pressure, plasma lipids and inflammatory markers: A randomised controlled trial. Eur J Nutr 2017;56:1421-1432.

115. de Bock M, Derraik JGB, Brennan CM, et al. Olive (Olea europaea L.) leaf polyphenols improve insulin sensitivity in middle-aged overweight men: A randomized, placebo-controlled, crossover trial. PLoS One 2013;8:e57622.

116. Wainstein J, Ganz T, Boaz M, et al. Olive leaf extract as a hypoglycemic agent in both human diabetic subjects and in rats. J Med Food 2012;15:605-610.

Khara Lucius, ND, FABNO, is a naturopathic doctor at the Center for Integrative Medicine, University of Pittsburgh Medical Center, Pittsburgh, Pennsylvania.

To order reprints of this article, contact the publisher at (914) 740-2100. 